\title{
Progress in Soft X-ray Microscopy Characterization of PEM Fuel Cell Catalyst Layers
} A. P. Hitchcock, ${ }^{1}$ J. Wu ${ }^{1}$, V. Lee, ${ }^{1}$ N. Appathurai, ${ }^{1}$ T. Tyliszczak $^{2}$, H.-W. Shiu, ${ }^{2}$ D.A. Shapiro,
V. Berejnov, ${ }^{3}$ D. Susac

1. Department of Chemistry and Chemical Biology - McMaster University, Hamilton, ON. Canada

${ }^{2}$ Advanced Light Source, LBNL, Berkeley, CA, USA

3. AFCC Automotive Fuel Cell Cooperation Corp., Burnaby, BC, Canada

Low temperature, hydrogen-fueled, proton exchange membrane fuel cell (PEM-FC) based engines are being developed rapidly for near-term implementation in mass production, personal automobiles. Materials and process research aiming to further optimize these systems is focused on understanding and controlling various degradation processes (carbon corrosion, Pt migration, cold start), and reducing cost by (i) reducing or eliminating Pt in the electro-catalyst, and (ii) optimizing the nanoscale distribution of the cathode components, in particular the proton conducting ionomer, to make most efficient use of the catalyst. Soft X-ray scanning transmission X-ray microscopy (STXM) [1] is a powerful tool to study PEM-FC catalyst layers (membrane electrode assemblies, MEA). STXM provides spectroscopic identification and quantitative mapping of chemical components with 20-30 nm spatial resolution in both $2 \mathrm{D}$ projection and 3D spectro-tomography. For a given radiation dose, it can provide better spectroscopic chemical characterization than analytical transmission electron microscopy (TEM) using electron energy loss or X-ray fluorescence methods. Given the very high sensitivity of the perfluorosulfonic acid (PFSA) membrane and ionomer to radiation damage [4], there are significant advantages to using X-ray microscopy for mapping ionomer in MEAs [2, 3]. An example of quantitative mapping of ionomer in catalyst layers in 2D projection by STXM is presented in Fig. 1. Recently, soft X-ray ptychography has been developed by Shapiro et al. at the ALS, with outstanding results on Li battery materials $[5,6]$. In collaboration with the ALS team, we are exploring how ptychography can provide improved spatial resolution for mapping ionomer in catalyst layers [7] (see Fig. 2). We are also measuring the 3D distributions of ionomer in catalyst layers by STXM spectro-tomography [8] and ptychography-based spectro-tomography. Progress is also being made on a feedback controlled variable humidity and temperature environmental cell to allow in situ studies of PEM-FC MEA under conditions similar to normal operation (e.g. $80 \mathrm{C}, 80 \% \mathrm{RH}$ ) or under cold start conditions (-30 C) (see Fig. 3).

This presentation will describe the instrumentation, methodology and data analysis involved in applying STXM and ptychography to PEM-FC catalyst layers; discuss the challenges and limitations; and illustrate their capabilities with results from studies of carbon corrosion [9], and alternate catalyst layer structures [8], as well as more recent method development work [10].

References:

[1] A.P. Hitchcock, Soft X-ray Imaging and Spectromicroscopy in Handbook on Nanoscopy, eds. G. Van Tendeloo, D. Van Dyck and S. J. Pennycook 2012. (Wiley)

[2] D. Susac et al, ECS Transactions 41 (2011), p. 629.

[3] D. Susac, V. Berejnov, A.P. Hitchcock and J. Stumper, ECS Transactions 50 (2012), p. 405.

[4] L. G. de A. Melo et al, Microscopy \& Microanalysis 21 S-3 (2015), p. 2443; J. Power Sources (2016) in press.

[5] D.A. Shapiro et al, Nature Photonics 8 (2014), p. 765.

[6] Y.S. Yu et al, Nano Letters 15 (2015), p. 4282. 
[7] A.P. Hitchcock, J. Electron Spectrosc. Rel. Phen. 200 (2015), p. 49.

[8] G. Schmid, et al, Book chapter in Characterization Tools for Nanoscience \& Nanotechnology, 5 (2015)

(Springer, Berlin, 2015)

[7] A.P. Hitchcock et al, J. Power Sources 266 (2014), p. 66.

[8] V. Lee et al, J. Power Sources 263 (2014), p. 163.

[9] STXM is performed on BL 10ID1 at the Canadian Light Source (CLS). CLS is supported by NSERC,CIHR, NRC and U. Saskatchewan. STXM is also performed on BL 5.3.2.2 at the Advanced Light Source (ALS). Ptychography is performed on BL 5.3.2.1 and 11.0.2 at the ALS. ALS is supported by Office of Basic Energy Science, Department of Energy. Ptychography processing performed using SHARP, a program of the Center for Applied Mathematics for Energy Research Applications, LBNL. Research supported by AFCC, NSERC, Canada Research Chairs, and the Catalyst Research for Polymer Electrolyte Fuel Cells (CaRPE-FC) network.
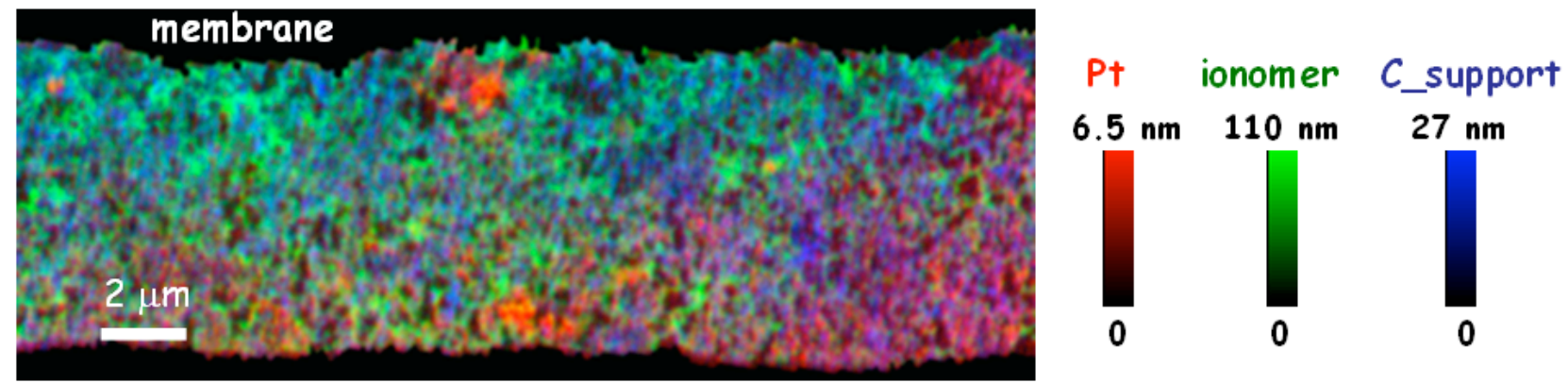

Figure 1: Color composite of quantitative component maps of Pt catalyst, ionomer and carbon support, in the cathode of a membrane electrode assembly (MEA) (BL 5322, ALS).

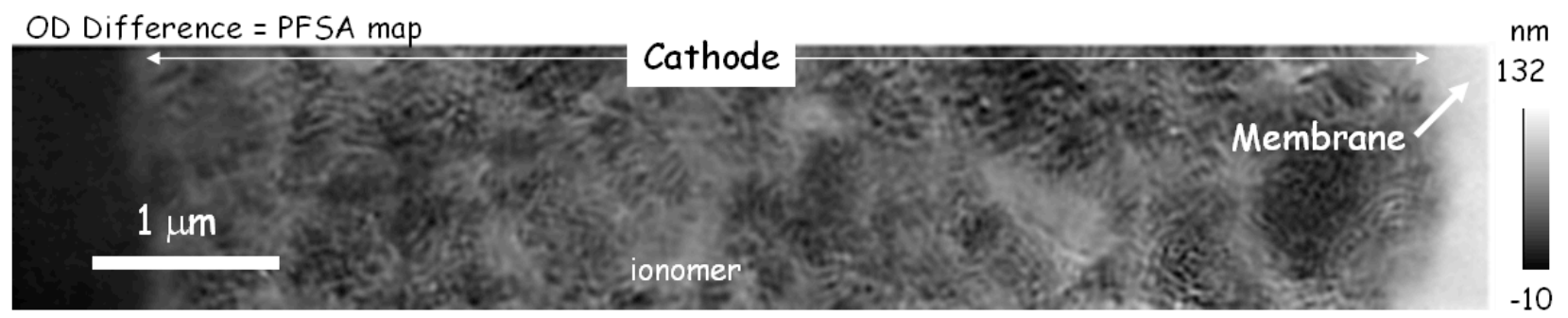

Figure 2: 2D projection image of PFSA in an MEA measured using spectro-ptychography. This image is the difference of ptychographic amplitude images recorded below $(683 \mathrm{eV})$ and above $(693 \mathrm{eV})$ the $\mathrm{F} 1 \mathrm{~s}$ absorption edge. The bright signal at the right is the membrane. (BL1102, ALS)
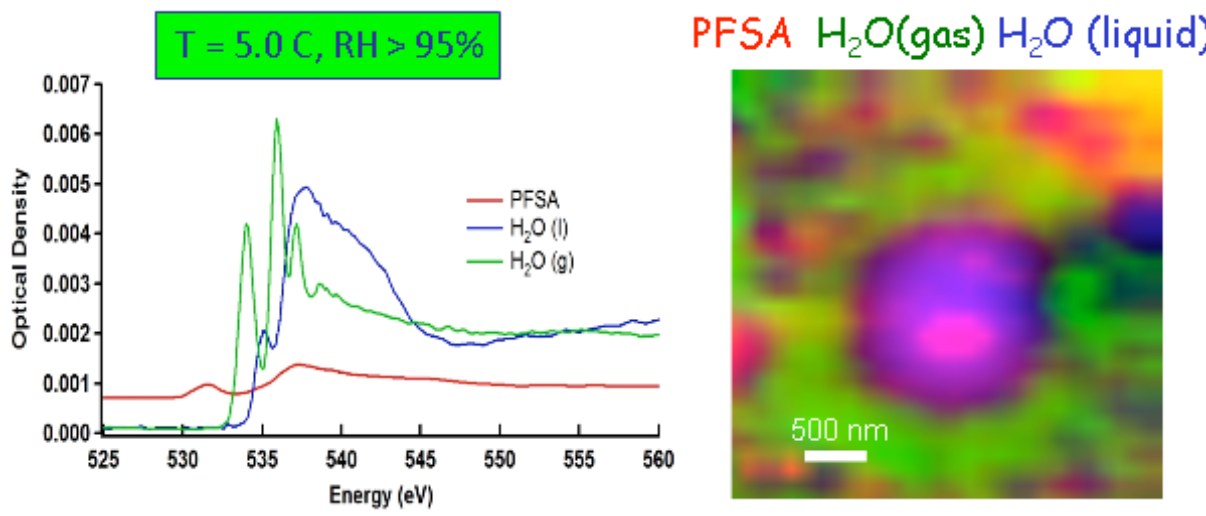

Figure 3: (left) $\mathrm{O} 1 \mathrm{~s}$ reference spectra used to fit a multi-image sequence at the $\mathrm{O} 1 \mathrm{~s}$ edge. (right) Color coded map of liquid water (blue), gaseous water (green) and PFSA (red) in an MEA measured by STXM under in situ conditions of $5 \mathrm{C}$ and 95\% RH. (BL 10ID1, CLS) 